Bull. Korean Math. Soc. 48 (2011), No. 1, pp. 141-149

DOI 10.4134/BKMS.2011.48.1.141

\title{
SOME TOEPLITZ OPERATORS ON WEIGHTED BERGMAN SPACES
}

\author{
Si Ho KANG
}

\begin{abstract}
We consider the problem to determine when a Toeplitz operator is bounded on weighted Bergman spaces. We show that Toeplitz operators induced by elements of some set are bounded and each element of the set is related with a Carleson measure on the weighted Bergman space.
\end{abstract}

\section{Introduction}

Let $d A$ denote normalized Lebesgue area measure on the unit disk $\mathbb{D}$. For $\alpha>-1$, the weighted Bergman space $A_{\alpha}^{2}$ consists of the analytic functions in $L^{2}\left(\mathbb{D}, d A_{\alpha}\right)$, where $d A_{\alpha}(z)=(\alpha+1)\left(1-|z|^{2}\right)^{\alpha} d A(z)$. Then $A_{\alpha}^{2}$ is closed in $L^{2}\left(\mathbb{D}, d A_{\alpha}\right)$ and for each $z \in \mathbb{D}$, there is a reproducing kernel $K_{z}^{\alpha}$ in $A_{\alpha}^{2}$ such that $f(z)=\left\langle f, K_{z}^{\alpha}\right\rangle$ for all $f \in A_{\alpha}^{2}$, in fact, $K_{z}^{\alpha}(w)=\frac{1}{(1-\bar{z} w)^{2+\alpha}}$ and the normalized reproducing kernel $k_{z}^{\alpha}$ is the function $\frac{K_{z}^{\alpha}}{\left\|K_{z}^{\alpha}\right\|_{2, \alpha}}$, that is, $k_{z}^{\alpha}(w)=\frac{\left(1-|z|^{2}\right)^{1+\frac{\alpha}{2}}}{(1-\bar{z} w)^{2+\alpha}}$, where the norm \|\|$_{2, \alpha}$ and the inner product are taken in the space $L^{2}\left(\mathbb{D}, d A_{\alpha}\right)$.

A linear operator $S$ on $A_{\alpha}^{2}$ induces a function $\widetilde{S}$ on $\mathbb{D}$ given by $\widetilde{S}(z)=$ $\left\langle S k_{z}^{\alpha}, k_{z}^{\alpha}\right\rangle, z \in \mathbb{D}$. The function $\widetilde{S}$ is called the Berezin transform of $S$.

For $u \in L^{1}(\mathbb{D}, d A)$, the Toeplitz operator $T_{u}^{\alpha}$ with symbol $u$ is the operator on $A_{\alpha}^{2}$ defined by $T_{u}^{\alpha}(f)=P_{\alpha}(u f), f \in A_{\alpha}^{2}$, where $P_{\alpha}$ is the orthogonal projection from $L^{2}\left(\mathbb{D}, d A_{\alpha}\right)$ onto $A_{\alpha}^{2}$ and let $\widetilde{u}$ denote $\widetilde{T_{u}^{\alpha}}$. Then the Toeplitz operator $T_{u}^{\alpha}$ is bounded whenever $u \in L^{\infty}(\mathbb{D}, d A)$ but every element of $L^{1}(\mathbb{D}, d A)$ dose not imply the boundness of the Toeplitz operator $T_{u}^{\alpha}$. Since $L^{\infty}(\mathbb{D}, d A)$ is dense in $L^{1}(\mathbb{D}, d A), T_{u}^{\alpha}$ is densely defined on $A_{\alpha}^{2}$. We note that Berezin transforms and Carleson measures are useful tools in the study of Toeplitz operators ([2], [4], $[5])$. Using those tools, many mathematicians working in operator theory are characterized the boundedness and compactness of Toeplitz operators.

Received May 26, 2009; Revised February 25, 2010.

2010 Mathematics Subject Classification. 47A15 and 47B35.

Key words and phrases. weighted Bergman spaces, Toeplitz operators, Berezin transforms, integral operators.

This research was partially supported by Sookmyung Women's University Research Grants 2009. 
In this paper, we prove that Toeplitz operators with special symbols are bounded and $\left\|u k_{z}^{\alpha}\right\|_{p, \alpha}$ having vanishing property implies the compactness of the Toeplitz operator $T_{u}^{\alpha}$.

Section 3 contains some properties of special symbols, that is, each element of some set implies a Carleson measure and we deal with appropriate products of Toeplitz operators and Hankel operators.

\section{Unitary operator and example}

Let $\operatorname{Aut}(\mathbb{D})$ denote the set of all bianalytic maps of $\mathbb{D}$ onto $\mathbb{D}$. By Schwarz's lemma, each element of Aut $(\mathbb{D})$ is a linear fractional transformation of the form $\lambda \varphi_{z},|\lambda|=1$, where $\varphi_{z}(w)=\frac{z-w}{1-\bar{z} w}, w \in \mathbb{D}$. Then $\varphi_{z} \circ \varphi_{z}$ is the identity function on $\mathbb{D}$ and $\operatorname{Aut}(\mathbb{D})$ is called the Möbius group under composition. For $\alpha>-1$ and $z \in \mathbb{D}$, we define $U_{z}^{\alpha} f(w)=f \circ \varphi_{z}(w) \frac{\left(1-|z|^{2}\right)^{\frac{\alpha}{2}+1}}{(1-\bar{z} w)^{\alpha+2}}, f \in L^{2}\left(\mathbb{D}, d A_{\alpha}\right), w \in \mathbb{D}$. A simple compactation shows that $U_{z}^{\alpha}$ is an isometry. Since $\left(1-\bar{z} \varphi_{z}(w)\right)^{\alpha+2}=$ $\left(\frac{1-|z|^{2}}{1-\bar{z} w}\right)^{\alpha+2}, U_{z}^{\alpha} U_{z}^{\alpha}$ is the identity operator and hence $\left(U_{z}^{\alpha}\right)^{*}=\left(U_{z}^{\alpha}\right)^{-1}=U_{z}^{\alpha}$, that is, $U_{z}^{\alpha}$ is a self-adjoint unitary operator on $A_{\alpha}^{2}$. Moreover, $U_{z}^{\alpha}\left(A_{\alpha}^{z}\right)=A_{\alpha}^{2}$ and $\left.U_{z}^{\alpha}\right|_{A_{\alpha}^{2}}$ is also denoted by $U_{z}^{\alpha}$ and $U_{z}^{\alpha} 1=k_{z}^{\alpha}(w)$.

For a linear operator $S$ on $A_{\alpha}^{2}$, we define the conjugate operator $S_{z}$ by $U_{z}^{\alpha} S U_{z}^{\alpha}$.

Now we are ready to state useful properties.

Lemma 2.1. For $u \in L^{1}(\mathbb{D}, d A)$ and $z \in \mathbb{D},\left(T_{u}^{\alpha}\right)_{z}=T_{u \circ \varphi_{z}}^{\alpha}$.

Proof. Since $\left(T_{u}^{\alpha}\right)_{z}=U_{z}^{\alpha} T_{u}^{\alpha} U_{z}^{\alpha}$ and $\left(U_{z}^{\alpha}\right)^{-1}=U_{z}^{\alpha}$, it is enough to show that $U_{z}^{\alpha} T_{u}^{\alpha}=T_{u \circ \varphi_{z}}^{\alpha} U_{z}^{\alpha}$. Take any $f$ in $A_{\alpha}^{2}$ and any $w$ in $\mathbb{D}$. Then

$$
\begin{aligned}
& U_{z}^{\alpha} T_{u}^{\alpha}(f)(w) \\
= & U_{z}^{\alpha} P_{\alpha}(u f)(w) \\
= & P_{\alpha}(u f)\left(\varphi_{z}(w)\right) \frac{\left(1-|z|^{2}\right)^{\frac{\alpha}{2}+1}}{(1-\bar{z} w)^{\alpha+2}} \\
= & (\alpha+1) \int_{\mathbb{D}} \frac{u(t) f(t)\left(1-|t|^{2}\right)^{\alpha}}{\left(1-\varphi_{z}(w) \bar{t}\right)^{2+\alpha}} d A(t) \frac{\left(1-|z|^{2}\right)^{\frac{\alpha}{2}+1}}{(1-\bar{z} w)^{\alpha+2}} \\
= & (\alpha+1) \int_{\mathbb{D}} u(t) f(t)\left(1-|t|^{2}\right)^{\alpha} \frac{(1-\bar{z} w)^{2+\alpha}}{(1-\bar{z} w-z \bar{t}+w \bar{t})^{2+\alpha}} d A(t) \frac{\left(1-|z|^{2}\right)^{\frac{\alpha}{2}+1}}{(1-\bar{z} w)^{\alpha+2}} \\
= & (\alpha+1) \int_{\mathbb{D}} u(t) f(t)\left(1-|t|^{2}\right)^{\alpha} \frac{\left(1-|z|^{2}\right)^{\frac{\alpha}{2}+1}}{(1-\bar{z} w-z \bar{t}+w \bar{t})^{2+\alpha}} d A(t) \\
= & (\alpha+1) \int_{\mathbb{D}} u \circ \varphi_{z}(s) f \circ \varphi_{z}(s)\left(1-\left|\varphi_{z}(s)\right|^{2}\right)^{\alpha} \frac{\left(1-|z|^{2}\right)^{\frac{\alpha}{2}+1}\left|\varphi_{z}^{\prime}(s)\right|^{2} d A(s)}{\left(1-\bar{z} w-z \overline{\varphi_{z}(s)}+w \overline{\varphi_{z}(s)}\right)^{2+\alpha}}
\end{aligned}
$$




$$
\begin{aligned}
= & (\alpha+1) \int_{\mathbb{D}} u \circ \varphi_{z}(s) f \circ \varphi_{z}(s) \frac{\left(1-|z|^{2}\right)^{\alpha}\left(1-|s|^{2}\right)^{\alpha}}{|1-\bar{z} s|^{2 \alpha}}\left(1-|z|^{2}\right)^{\frac{\alpha}{2}+1} \frac{\left|\left(1-|z|^{2}\right)\right|^{2}}{|1-\bar{z} s|^{4}} \\
& \times \frac{(1-z \bar{s})^{2+\alpha} d A(s)}{\left(1-|z|^{2}\right)^{2+\alpha}(1-w \bar{s})^{2+\alpha}} \\
= & (\alpha+1) \int_{\mathbb{D}} u \circ \varphi_{z}(s) f \circ \varphi_{z}(s) \frac{\left(1-|s|^{2}\right)^{\alpha}\left(1-|z|^{2}\right)^{\frac{\alpha}{2}+1}(1-z \bar{s})^{2+\alpha} d A(s)}{(1-\bar{z} s)^{\alpha}(1-z \bar{s})^{\alpha}(1-\bar{z} s)^{2}(1-z \bar{s})^{2}(1-w \bar{s})^{2+\alpha}} \\
= & (\alpha+1) \int_{\mathbb{D}} u \circ \varphi_{z}(s) f \circ \varphi_{z}(s) \frac{\left(1-|z|^{2}\right)^{\frac{\alpha}{2}+1}\left(1-|s|^{2}\right)^{\alpha}}{(1-\bar{z} s)^{\alpha+2}(1-w \bar{s})^{2+\alpha}} d A(s) \\
= & \int_{\mathbb{D}} u \circ \varphi_{z}(s) U_{z}^{\alpha} f(s) \frac{d A_{\alpha}(s)}{(1-w \bar{s})^{2+\alpha}} \\
= & P_{\alpha}\left(u \circ \varphi_{z} U_{z}^{\alpha} f\right)(w) .
\end{aligned}
$$

$\operatorname{Thus}\left(T_{u}^{\alpha}\right)_{z}=T_{u \circ \varphi_{z}}^{\alpha}$.

Corollary 2.2. If $u_{1}, \ldots, u_{n} \in L^{1}(\mathbb{D}, d A)$, then

$$
U_{z}^{\alpha} T_{u_{1}} \cdots T_{u_{n}} U_{z}^{\alpha}=T_{u_{1} \circ \varphi_{z}} \cdots T_{u_{n} \circ \varphi_{z}} .
$$

Proof. It follows from the fact that $U_{z}^{\alpha} U_{z}^{\alpha}$ is the identity.

Proposition 2.3. For $u \in L^{1}(\mathbb{D}, d A)$ and $z \in \mathbb{D}, \widetilde{T_{u}^{\alpha}} \circ \varphi_{z}=\left(\widetilde{T_{u}^{\alpha}}\right)_{z}$.

Proof. Since $\widetilde{\left(T_{u}^{\alpha}\right)_{z}}=\widetilde{T_{u \circ \varphi_{z}}^{\alpha}}$, it is enough to show that $\widetilde{T_{u \circ \varphi_{z}}^{\alpha}}=\widetilde{T_{u}^{\alpha} \circ \varphi_{z}}$.

Take any $t$ in $\mathbb{D}$. Then

$$
\begin{aligned}
& \widetilde{T}_{u}^{\alpha} \circ \varphi_{z}(t) \\
= & \left\langle T_{u}^{\alpha} k_{\varphi_{z}(t)}^{\alpha}, k_{\varphi_{z}(t)}^{\alpha}\right\rangle \\
= & \left\langle P_{\alpha}\left(u k_{\varphi_{z}(t)}^{\alpha}\right), k_{\varphi_{z}(t)}^{\alpha}\right\rangle \\
= & \left\langle u k_{\varphi_{z}(t)}^{\alpha}, k_{\varphi_{z}(t)}^{\alpha}\right\rangle \\
= & \int_{\mathbb{D}} u(x) k_{\varphi_{z}(t)}^{\alpha}(x) \overline{k_{\varphi_{z}(t)}^{\alpha}(x)} d A_{\alpha}(x) \\
= & \int_{\mathbb{D}} u(x)\left(\frac{\left(1-|z|^{2}\right)\left(1-|t|^{2}\right)}{|1-\bar{z}|^{2}}\right)^{1+\frac{\alpha}{2}}\left(\frac{1-z \bar{t}}{1-z \bar{t}-\bar{z} x+\bar{t} z}\right)^{2+\alpha}\left(\frac{\left(1-|z|^{2}\right)\left(1-|t|^{2}\right)}{|1-\bar{z} t|^{2}}\right)^{1+\frac{\alpha}{2}} \\
& \times\left(\frac{1-\bar{z} t}{1-\bar{z} t-z \bar{x}+t \bar{x}}\right)^{2+\alpha}(\alpha+1)\left(1-|x|^{2}\right)^{\alpha} d A(x) \\
= & \int_{\mathbb{D}} u(x)\left(1-|t|^{2}\right)^{1+\frac{\alpha}{2}}\left(\frac{1}{(1-\bar{z} t)(1-z \bar{t})}\right)^{1+\frac{\alpha}{2}}\left(\frac{1-z \bar{t}}{1-z \bar{t}-\bar{z} x+\bar{t} x}\right)^{2+\alpha}\left(1-|t|^{2}\right)^{1+\frac{\alpha}{2}} \\
& \times\left(1-|z|^{2}\right)^{2+\alpha} \frac{1+\frac{\alpha}{2}}{(1-\bar{z} t)(1-z \bar{t})}\left(\frac{1-\bar{z} t}{1-\bar{z} t-z \bar{x}+t \bar{x}}\right)^{2+\alpha}(\alpha+1)\left(1-|x|^{2}\right)^{\alpha} d A(x) \\
& \times\left(\frac{1}{(1+\alpha}\right)
\end{aligned}
$$




$$
\begin{aligned}
= & \int_{\mathbb{D}} u(x)\left(1-|t|^{2}\right)^{1+\frac{\alpha}{2}}\left(\frac{1-\bar{z} x}{1-\bar{z} x-\bar{t} z+\bar{t} x}\right)^{2+\alpha}\left(1-|t|^{2}\right)^{1+\frac{\alpha}{2}}\left(\frac{1-z \bar{x}}{1-z \bar{x}-t \bar{z}+t \bar{x}}\right)^{2+\alpha} \\
& \times \frac{\left(1-|z|^{2}\right)^{\alpha+2}}{|1-\bar{z} x|^{2 \alpha+4}} d A_{\alpha}(x) \\
= & \int_{\mathbb{D}} u \circ \varphi_{z}(s) k_{t}^{\alpha}(s) \overline{k_{t}^{\alpha}(s)} d A_{\alpha}(s) \\
= & \left\langle u \circ \varphi_{z} k_{t}^{\alpha}, k_{t}^{\alpha}\right\rangle \\
= & \left\langle P_{\alpha}\left(u \circ \varphi_{z} k_{t}^{\alpha}\right), k_{t}^{\alpha}\right\rangle \\
= & \left\langle T_{u \circ \varphi_{z}}^{\alpha} k_{t}^{\alpha}, k_{t}^{\alpha}\right\rangle \\
= & \widetilde{T_{u \circ \varphi_{z}}^{\alpha}}(t) .
\end{aligned}
$$

This completes the proof.

We will show that the Toeplitz operators with special symbols are bounded. To do so, we need the following proposition, in fact, the following proposition holds for every linear operator on $A_{\alpha}^{2}$.

Proposition 2.4. If $S$ is a linear operator on $A_{\alpha}^{2}$ and $z, w \in \mathbb{D}$, then $\left(S_{z}\right)^{*}=$ $\left(S^{*}\right)_{z}$ and $S K_{z}^{\alpha}(w)=\overline{S^{*} K_{w}^{\alpha}(z)}$.

Proof. Take any $f, g$ in $A_{\alpha}^{2}$. Since $\left\langle S_{z} f, g\right\rangle=\left\langle U_{z}^{\alpha} S U_{z}^{\alpha} f, g\right\rangle=\left\langle f, U_{z}^{\alpha} S^{*} U_{z}^{\alpha} g\right\rangle=$ $\left\langle f,\left(S^{*}\right)_{z} g\right\rangle,\left(S_{z}\right)^{*}=\left(S^{*}\right)_{z}$. For the 2nd equality, $S K_{z}^{\alpha}(w)=\left\langle S K_{z}^{\alpha}, K_{w}^{\alpha}\right\rangle=$ $\left\langle K_{z}^{\alpha}, S^{*} K_{w}^{\alpha}\right\rangle=\overline{S^{*} K_{w}^{\alpha}(z)}$

Since $K_{z}^{\alpha}(w)=\frac{1}{(1-\bar{z} w)^{2+\alpha}}=\sum_{n=0}^{\infty} \frac{\Gamma(n+2+\alpha)}{n ! \Gamma(2+\alpha)} \bar{z}^{n} w^{n}, k_{z}^{\alpha}(w)=\left(1-|z|^{2}\right)^{1+\frac{\alpha}{2}} \times$ $\sum_{n=0}^{\infty} \frac{\Gamma(n+2+\alpha)}{n ! \Gamma(2+\alpha)} \bar{z}^{n} w^{n}$. We define $S\left(\sum a_{n} w^{n}\right)=\sum a_{n}(-w)^{n}$. Then $S\left(K_{z}^{\alpha}(w)\right)$ $=\sum_{n=0}^{\infty} \frac{\Gamma(n+2+\alpha)}{n ! \Gamma(2+\alpha)} \bar{z}^{n}(-1)^{n} w^{n}=K_{z}^{\alpha}(-w)$ and $S$ has an infinite-dimensional range and $S$ is an isometry and invertible, that is, $S^{*}=S^{-1}=S$. Thus $S$ is not compact. Since $\widetilde{S}(z)=\left\langle S k_{z}^{\alpha}, k_{z}^{\alpha}\right\rangle=\left(1-|z|^{2}\right)^{2+\alpha}\left\langle S K_{z}^{\alpha}, K_{z}^{\alpha}\right\rangle=$ $\left(1-|z|^{2}\right)^{2+\alpha} K_{z}^{\alpha}(-z)=\left(\frac{1-|z|^{2}}{1+|z|^{2}}\right)^{2+\alpha}, \lim _{z \rightarrow \partial \mathbb{D}} \widetilde{S}(z)=0$ and hence the vanishing property does not imply the compactness of operators.

\section{Toeplitz operators with special symbols}

This section deals with Toeplitz operators with special symbols. We begin by constructing some set and show that each element of the set implies a bounded linear operator. Recall that $P_{\alpha}$ is the orthogonal projection from $L^{2}\left(\mathbb{D}, d A_{\alpha}\right)$ onto $A_{\alpha}^{2}$ and for $z \in \mathbb{D}$ and $f \in L^{2}\left(\mathbb{D}, d A_{\alpha}\right), P_{\alpha}(f)(z)=\left\langle P_{\alpha}(f), K_{z}^{\alpha}\right\rangle=$ $\int_{\mathbb{D}} f(w) \overline{K_{z}^{\alpha}(w)} d A_{\alpha}(w)$. Moreover, we extend the domain of $P_{\alpha}$ to $L^{1}\left(\mathbb{D}, d A_{\alpha}\right)$ and for $f \in A_{\alpha}^{1}, f(z)=\int_{\mathbb{D}} \frac{f(w) d A_{\alpha}(w)}{(1-z \bar{w})^{2+\alpha}}, z \in \mathbb{D}$ (see [5]). 
Let $M K=\left\{u \in L^{1}(\mathbb{D}, d A): \sup _{\lambda \in \mathbb{D}}\left\|u k_{\lambda}^{\alpha}\right\|_{p, \alpha}<\infty\right.$ for every $\left.p \in(1, \infty)\right\}$, where $\|\cdot\|_{p, \alpha}$ is the norm on $L^{p}\left(\mathbb{D}, d A_{\alpha}\right)$. Since $\left\|u k_{\lambda}\right\|_{p, \alpha}=\left\|\bar{u} k_{\lambda}\right\|_{p, \alpha}, M K$ is closed under the formation of conjugations.

Lemma 3.1. For any $u \in M K,\left(T_{u}^{\alpha}\right)^{*}=T_{\bar{u}}^{\alpha}$.

Proof. Take any $f, g$ in $A_{\alpha}^{2}$. Since $\left\langle T_{u}^{\alpha} f, g\right\rangle=\left\langle P_{\alpha}(u f), g\right\rangle=\langle u f, g\rangle=\langle f, \bar{u} g\rangle=$ $\left\langle f, P_{\alpha}(\bar{u} g)\right\rangle=\left\langle f, T_{\bar{u}}^{\alpha} g\right\rangle,\left(T_{u}\right)^{*}=T_{\bar{u}}^{\alpha}$.

Lemma 3.2. Suppose $u \in M K, z \in \mathbb{D}$, and $p \in(1, \infty)$. Then there is a constant $c>0$ such that $\left\|\left(T_{u}^{\alpha}\right)_{z} 1\right\|_{p, \alpha} \leq c\left\|u k_{z}^{\alpha}\right\|_{p, \alpha}$.

Proof. Since $\left(T_{u}^{\alpha}\right)_{z}=U_{z}^{\alpha} T_{u}^{\alpha} U_{z}^{\alpha}$ and $U_{z}^{\alpha} 1=k_{z}^{\alpha},\left\|\left(T_{u}^{\alpha}\right)_{z} 1\right\|_{p, \alpha}=\left\|U_{z}^{\alpha} T_{u}^{\alpha} U_{z}^{\alpha} 1\right\|_{p, \alpha}$ $=\left\|U_{z}^{\alpha} P_{\alpha}\left(u k_{z}^{\alpha}\right)\right\|_{p, \alpha} \leq\left\|P_{\alpha}\right\|\left\|u k_{z}^{\alpha}\right\|_{p, \alpha}$ and hence $\left\|\left(T_{u}^{\alpha}\right)_{z} 1\right\|_{p, \alpha} \leq c\left\|u k_{z}^{\alpha}\right\|_{p, \alpha}$ for some $c$.

Suppose $f \in A_{\alpha}^{2}$ and $w \in \mathbb{D}$. Then $\left(T_{u}^{\alpha} f\right)(w)=\left\langle T_{u}^{\alpha} f, K_{w}^{\alpha}\right\rangle=\left\langle f,\left(T_{u}^{\alpha}\right)^{*} K_{w}^{\alpha}\right\rangle$ $=\int_{\mathbb{D}} f(z) \overline{\left(T_{u}^{\alpha}\right)^{*} K_{w}^{\alpha}(z)} d A_{\alpha}(z)=\int_{\mathbb{D}} f(z)\left(T_{u}^{\alpha} K_{z}^{\alpha}\right)(w) d A_{\alpha}(z)$, here the last equality follows from Proposition 2.4. Thus $T_{u}^{\alpha}$ is the integral operator with kernel $\left(T_{u}^{\alpha} K_{z}^{\alpha}\right)(w)$.

Lemma 3.3. Suppose $0<t, s \in(1, \infty)$ and $u \in M K$. Then there is a constant $c>0$ such that $\int_{\mathbb{D}} \frac{\left|\left(T_{u}^{\alpha} K_{z}^{\alpha}\right)(w)\right|}{\left(1-|w|^{2}\right)^{t}} \leq \frac{c\left\|u k_{z}^{\alpha}\right\|_{s, \alpha}}{\left(1-|z|^{2}\right)^{t}}$ for all $z \in \mathbb{D}$ and $\int_{\mathbb{D}} \frac{\left|\left(T_{u}^{\alpha} K_{z}^{\alpha}\right)(w)\right|}{\left(1-|z|^{2}\right)^{t}} d A_{\alpha}(z) \leq \frac{c\left\|\bar{u} k_{w}^{\alpha}\right\|_{s, \alpha}}{\left(1-|w|^{2}\right)^{t}}$ for all $w \in \mathbb{D}$.

Proof. Since $k_{z}^{\alpha}=\left(1-|z|^{2}\right)^{1+\frac{\alpha}{2}} K_{z}^{\alpha}, T_{u}^{\alpha} K_{z}^{\alpha}=\frac{U_{z}^{\alpha}\left(T_{u}^{\alpha}\right)_{z} 1}{\left(1-|z|^{2}\right)^{1+\frac{\alpha}{2}}}=\frac{\left(T_{u}^{\alpha}\right) 10 \varphi_{z} \frac{\left(1-|z|^{2}\right)^{\frac{\alpha}{2}+1}}{(1-\bar{z})^{\alpha+2}} z}{\left(1-|z|^{2}\right)^{1+\frac{\alpha}{2}}}$ and hence

$$
\begin{aligned}
& \int_{\mathbb{D}} \frac{\mid\left(\left(T_{u}^{\alpha} K_{z}^{\alpha}\right)(w) \mid\right.}{\left(1-|w|^{2}\right)^{t}} d A_{\alpha}(w) \\
= & \int_{\mathbb{D}} \frac{\left|\left(\left(T_{u}^{\alpha}\right)_{z} 1\right) \circ \varphi_{z}(w)\right| \frac{\left(1-|z|^{2}\right)^{\frac{2}{\alpha}+1}}{|1-\bar{z} w|^{\alpha+2}}\left(1-|w|^{2}\right)^{\alpha} d A(w)}{\left(1-|z|^{2}\right)^{\frac{\alpha}{2}+1}\left(1-|w|^{2}\right)^{t}} \\
= & \int_{\mathbb{D}} \frac{\left|\left(\left(T_{u}^{\alpha}\right)_{z} 1\right)(\lambda)\right| \frac{1}{\left|1-\bar{z} \varphi_{z}(\lambda)\right|^{\alpha+2}}}{\left(1-\left|\varphi_{z}(\lambda)\right|^{2}\right)^{t}}\left(1-\left|\varphi_{z}(\lambda)\right|^{2}\right)^{\alpha}\left|\varphi_{z}^{\prime}(\lambda)\right|^{2} d A(\lambda) \\
= & \int_{\mathbb{D}} \frac{\left|\left(\left(T_{u}^{\alpha}\right)_{z} 1\right)(\lambda)\right| \frac{|1-\bar{z} \lambda|^{\alpha+2}}{\left(1-|z|^{2}\right)^{\alpha+2}}}{\frac{\left(1-|z|^{2}\right)^{t}\left(1-|\lambda|^{2}\right)^{t}}{|1-\bar{z} \lambda|^{2 t}}} \frac{\left(1-|z|^{2}\right)^{\alpha}\left(1-|\lambda|^{2}\right)^{\alpha}}{|1-\bar{z} \lambda|^{2 \alpha}} \frac{\left(1-|z|^{2}\right)^{2}}{|1-\bar{z} \lambda|^{4}} d A(\lambda) \\
= & \frac{1}{\left(1-|z|^{2}\right)^{t}} \int_{\mathbb{D}} \frac{\left|\left(\left(T_{u}^{\alpha}\right)_{z} 1\right)(\lambda)\right|}{|1-\bar{z} \lambda|^{2-2 t+\alpha}} \frac{1}{\left(1-|\lambda|^{2}\right)^{t-\alpha}} d A(\lambda) \\
\leq & \frac{1}{\left(1-|z|^{2}\right)^{t}}\left\|\left(T_{u}^{\alpha}\right)_{z} 1\right\|_{s, \alpha}\left(\int_{\mathbb{D}} \frac{1}{|1-\bar{z} \lambda|^{(2-2 t+\alpha) s^{\prime}}\left(1-|\lambda|^{2}\right)^{t s^{\prime}-\alpha}} d A(\lambda)\right)^{\frac{1}{s^{\prime}}},
\end{aligned}
$$


where $s$ and $s^{\prime}$ are conjugate exponents.

By Lemma 3.2, there is a constant $c_{1}$, such that $\left\|\left(T_{u}^{\alpha}\right)_{z} 1\right\|_{s, \alpha} \leq c_{1}\left\|u k_{z}^{\alpha}\right\|_{s, \alpha}$.

Let $c=c_{1}\left(\int_{\mathbb{D}} \frac{1}{|1-\bar{z} \lambda|^{(2-2 t+\alpha) s^{\prime}}\left(1-|\lambda|^{2}\right)^{t s^{\prime}-\alpha}} d A(\lambda)\right)^{\frac{1}{s^{\prime}}}$. If $c$ is infinity, then trivially the inequality holds and hence $\int_{\mathbb{D}} \frac{\left|\left(T_{u}^{\alpha} K_{z}^{\alpha}\right)(w)\right|}{\left(1-|w|^{2}\right)^{t}} d A_{\alpha}(w) \leq \frac{c\left\|u k_{z}^{\alpha}\right\|_{s, \alpha}}{\left(1-|z|^{2}\right)^{t}}$. Proposition 2.4 and Lemma 3.1 and $\overline{M K} \subset M K$ imply that there is a constant $c$ such that $\int_{\mathbb{D}} \frac{\left|\left(T_{u}^{\alpha} K_{z}^{\alpha}\right)(w)\right|}{\left(1-|z|^{2}\right)^{t}} d A_{\alpha}(z)=\int_{\mathbb{D}} \frac{\left|\left(T_{u}^{\alpha} K_{w}^{\alpha}\right)(z)\right|}{\left(1-|z|^{2}\right)^{t}} d A_{\alpha}(z) \leq \frac{c\left\|\bar{u} k_{w}^{\alpha}\right\|_{s, \alpha}}{\left(1-|w|^{2}\right)^{t}}$. This completes the proof.

Suppose $\alpha \neq 0$. Then there is $t>0$ such that $s^{\prime}=\frac{2 \alpha}{4 t-2-\alpha}>1$ and

$$
\int_{\mathbb{D}} \frac{1}{|1-\bar{z} \lambda|^{(2-2 t+\alpha) s^{\prime}}\left(1-|\lambda|^{2}\right)^{t s^{\prime}-\alpha}} d A(\lambda)=\int_{\mathbb{D}} \frac{1}{|1-\bar{z} \lambda|^{\frac{6}{5}}\left(1-|\lambda|^{2}\right)^{\frac{3}{5}}} d A(\lambda) .
$$

Axler's paper ([1], Lemma 4) asserts that the last integral is finite. In Lemma $3.3, c$ is finite.

Theorem 3.4. For each $u \in M K, T_{u}^{\alpha}$ is bounded.

Proof. Let $h(\lambda)=\frac{1}{\left(1-|\lambda|^{2}\right)^{t}}$. By the above observation and Lemma 3.3,

$$
\int_{\mathbb{D}} \frac{\left|\left(T_{u}^{\alpha} K_{z}^{\alpha}\right)(w)\right|}{\left(1-|w|^{2}\right)^{t}} d A_{\alpha}(w) \leq c_{1} h(z) \text { and } \int_{\mathbb{D}} \frac{\left|\left(T_{u}^{\alpha} K_{z}^{\alpha}\right)(w)\right|}{\left(1-|z|^{2}\right)^{t}} d A_{\alpha}(z) \leq c_{2} h(w),
$$

where $c_{1}=c \sup \left\|u k_{z}^{\alpha}\right\|_{s, \alpha}$ and $c_{2}=c \sup \left\|\bar{u} k_{w}^{\alpha}\right\|_{s, \alpha}$.

The Schur's test (see page 126 of [3]) implies that $T_{u}^{\alpha}$ is bounded and $\left\|T_{u}^{\alpha}\right\| \leq$ $\sqrt{c_{1} c_{2}}$.

Recall that $T_{u}^{\alpha}$ is the integral operator with kernel $\left(T_{u}^{\alpha} K_{z}^{\alpha}\right)(w)$, that is, $\left(T_{u}^{\alpha} f\right)(w)=\int_{\mathbb{D}} f(z)\left(T_{u}^{\alpha} K_{z}^{\alpha}\right)(w) d A(z)$. For $0<r<1$, we define an operator $T_{r}^{\alpha}$ on $A_{\alpha}^{2}$ by $\left(T_{r}^{\alpha} f\right)(w)=\int_{r \mathbb{D}} f(z) T_{u}^{\alpha} K_{z}^{\alpha}(w) d A(z), f \in A_{\alpha}^{2}$. Since $\int_{\mathbb{D}} \int_{\mathbb{D}}\left|T_{u}^{\alpha} K_{z}^{\alpha}(w) \chi_{r \mathbb{D}}(z)\right|^{2} d A_{\alpha}(w) d A_{\alpha}(z)=\int_{r \mathbb{D}} \int_{\mathbb{D}}\left|T_{u}^{\alpha} K_{z}^{\alpha}(w)\right|^{2} d A_{\alpha}(w) d A_{\alpha}(z)=$ $\int_{r \mathbb{D}}\left\|T_{u}^{\alpha} K_{z}^{\alpha}\right\|_{2}^{2} d A_{\alpha}(z) \leq\left\|T_{u}^{\alpha}\right\|^{2} \int_{r \mathbb{D}}\left\|K_{z}^{\alpha}\right\|_{2}^{2} d A(z)<\infty, T_{u}^{\alpha} K_{z}^{\alpha}(w) \chi_{r \mathbb{D}}(z) \in L^{2}(\mathbb{D} \times$ $\left.\mathbb{D}, d A_{\alpha} \times d A_{\alpha}\right)$ and hence $T_{r}^{\alpha}$ is a Hilbert-Schmidt operator. Thus each $T_{r}^{\alpha}$ is compact. Let $h(\lambda)=\left(\frac{1}{1-|\lambda|^{2}}\right)^{t}$. By Lemma 3.3 and Theorem 3.4,

$$
\begin{aligned}
& \int_{\mathbb{D}}\left|T_{u}^{\alpha} K_{z}^{\alpha}(w) \chi_{\mathbb{D} \backslash r \mathbb{D}}(z)\right| h(w) d A_{\alpha}(w) \leq c_{1} h(z) \text { and } \\
& \int_{\mathbb{D}}\left|T_{u}^{\alpha} K_{z}^{\alpha}(w) \chi_{\mathbb{D} \backslash r \mathbb{D}}(z)\right| h(z) d A_{\alpha}(z) \leq c_{2} h(w),
\end{aligned}
$$

where $c_{1}=c \sup _{r \leq|z|<1}\left\|u k_{z}^{\alpha}\right\|_{s, \alpha}$ and $c_{2}=c \sup \left\|\bar{u} k_{w}^{\alpha}\right\|_{s, \alpha}$. The Schur's test implies that $\left\|T_{u}^{\alpha}-T_{r}^{\alpha}\right\| \leq c_{1}^{\frac{1}{2}} c_{2}^{\frac{1}{2}}$. If $\lim _{z \rightarrow \partial \mathbb{D}}\left\|u k_{z}^{\alpha}\right\|_{s, \alpha}=0$, then $\lim _{r \rightarrow 1^{-}} c_{1}=0$ and hence $\lim _{r \rightarrow 1^{-}}\left\|T_{u}^{\alpha}-T_{r}^{\alpha}\right\|=0$. Since each $T_{r}^{\alpha}$ is compact, $T_{u}^{\alpha}$ is also a compact operator. Thus we have the following theorem. 
Theorem 3.5. Let $u \in M K$. If $\lim _{z \rightarrow \partial \mathbb{D}}\left\|u k_{z}^{\alpha}\right\|_{p, \alpha}=0$ for every $p \in(1, \infty)$, then $T_{u}^{\alpha}$ is a compact operator.

We note that $A_{\alpha}^{p}$ consists of the analytic functions in $L^{p}\left(\mathbb{D}, d A_{\alpha}\right)$. Suppose $\mu$ is a finite positive Borel measure on $\mathbb{D}$ and $p>1$. Recall that if $i_{p}: A_{\alpha}^{p} \rightarrow$ $L^{p}(\mathbb{D}, d \mu)$ is bounded, then $\mu$ is called a Carleson measure on the Bergman space $A_{\alpha}^{p}$ and Carleson measures are very useful tools in operator theory.

Proposition 3.6. For $u \in M K,|u| d A_{\alpha}$ is a Carleson measure on $A_{\alpha}^{p}$.

Proof. It is enough to show that $\widetilde{u}$ is bounded. For $w \in \mathbb{D},|\widetilde{u}(w)|=\left|\left\langle T_{u}^{\alpha} k_{w}^{\alpha}, k_{w}^{\alpha}\right\rangle\right|$ $=\left|\left\langle P_{\alpha}\left(u k_{w}^{\alpha}\right), k_{w}^{\alpha}\right\rangle\right|=\left|\left\langle u k_{w}^{\alpha}, k_{w}^{\alpha}\right\rangle\right| \leq\left\|u k_{w}^{\alpha}\right\|_{2, \alpha}<\infty$. Thus $|u| d A_{\alpha}$ is a Carleson measure on $A_{\alpha}^{p}$.

Corollary 3.7. For $u \in M K, T_{u}^{\alpha}$ is a bounded linear operator.

Proof. It follows from the fact that $|u| d A_{\alpha}$ is a Carleson measure.

Using the concept of a Carleson measure, we can give another proof for Theorem 3.5.

Proposition 3.8. If $\left\|u k_{z}^{\alpha}\right\|_{p, \alpha} \rightarrow 0$ as $z \rightarrow \partial \mathbb{D}$ for every $p \in(1, \infty)$ and $u \in M K$, then $T_{u}^{\alpha}$ is compact.

Proof. Let's show that $|u| d A_{\alpha}$ is a vanishing Carleson measure on the Bergman space $A_{\alpha}^{p}$. To do so, it is enough to show that $\lim _{|z| \rightarrow 1^{-}}|\widetilde{u}|(z)=0$. For $z \in$ $\mathbb{D},|\widetilde{u}(z)|=\left|\left\langle T_{u}^{\alpha} k_{z}^{\alpha}, k_{z}^{\alpha}\right\rangle\right|=\left|\left\langle u k_{z}^{\alpha}, k_{z}^{\alpha}\right\rangle\right| \leq\left\|u k_{z}^{\alpha}\right\|_{2, \alpha}\left\|k_{z}^{\alpha}\right\|_{2, \alpha}=\left\|u k_{z}^{\alpha}\right\|_{2, \alpha}$. The property $\lim _{|z| \rightarrow 1^{-}}\left\|u k_{z}^{\alpha}\right\|_{2, \alpha}=0$ implies that $|u| d A_{\alpha}$ is a vanishing Carleson measure. Thus $T_{|u|}^{\alpha}$ is compact. Since $\left.\left.\left|\int_{\mathbb{D}}\right| f_{u}\right|^{2} u d A_{\alpha}\left|\leq \int_{\mathbb{D}}\right| f_{u}\right|^{2}|u| d A_{\alpha}, T_{u}^{\alpha}$ is also compact.

We define an operator $H_{u}^{\alpha}: A_{\alpha}^{2} \rightarrow\left(A_{\alpha}^{2}\right)^{\perp}$ by

$$
H_{u}^{\alpha}(g)=\left(I-P_{\alpha}\right)(u g), g \in A_{\alpha}^{2} .
$$

Then $H_{u}^{\alpha}$ is called the Hankel operator on the weighted Bergman space with symbol $u$. Clearly $H_{u}^{\alpha}$ is densely defined for any $u \in L^{1}(\mathbb{D}, d A)$ and if $u \in$ $L^{\infty}(\mathbb{D}, d A)$, then $H_{u}^{\alpha}$ is bounded with $\left\|H_{u}^{\alpha}\right\| \leq\|u\|_{\infty}$.

Proposition 3.9. If $u^{2} \in M K$, then $H_{u}^{\alpha}$ is bounded.

Proof. Take any $f$ in $A_{\alpha}^{2}$. Then $\left\|H_{u}^{\alpha}(f)\right\|_{2, \alpha}^{2}=\left\|\left(I-P_{\alpha}\right)(u f)\right\|_{2, \alpha}^{2} \leq\|u f\|_{2, \alpha}^{2}=$ $\int_{\mathbb{D}}|f(z)|^{2}|u(z)|^{2} d A_{\alpha}(z)$. By Proposition 3.6, $|u|^{2} d A_{\alpha}$ is a Carleson measure on $A_{\alpha}^{2}$ and hence there is a constant $c<\infty$ such that $\int_{\mathbb{D}}|f(z)|^{2}|u(z)|^{2} d A_{\alpha}(z) \leq$ $c \int_{\mathbb{D}}|f(z)|^{2} d A_{\alpha}(z)$. Thus $H_{u}^{\alpha}$ is bounded.

Corollary 3.10. (1) Suppose $u^{2} \in M K$. Then $\left(H_{u}^{\alpha}\right)_{z} 1$ and $H_{u}^{\alpha} k_{z}^{\alpha}$ are in $L^{2}\left(\mathbb{D}, d A_{\alpha}\right)$ for every $z \in \mathbb{D}$.

(2) Suppose $u^{2} \in M K$ and $z \in \mathbb{D}$. Then $H_{u}^{\alpha} \circ \varphi_{z}$ is bounded. 
Proof. (1) We note that $\left\|H_{u}^{\alpha} U_{z}^{\alpha} 1\right\|_{2, \alpha}=\left\|H_{u}^{\alpha} k_{z}^{\alpha}\right\|_{2, \alpha}$ and hence $\left\|\left(H_{u}^{\alpha}\right)_{z} 1\right\|_{2, \alpha}=$ $\left\|H_{u}^{\alpha} k_{z}^{\alpha}\right\|_{2, \alpha}$. Since $\left\|\left(H_{u}^{\alpha}\right)_{z}\right\| 1_{2, \alpha}=\left\|U_{z}^{\alpha} H_{u}^{\alpha} U_{z}^{\alpha} 1\right\|_{2, \alpha}=\left\|H_{u}^{\alpha} k_{z}^{\alpha}\right\|_{2, \alpha} \leq\left\|H_{u}^{\alpha}\right\|$, we have the results.

(2) By Lemma 2.1, $\left(T_{u}^{\alpha}\right)_{z}=T_{u \circ \varphi_{z}}^{\alpha}$. Then $\left(H_{u}^{\alpha}\right)_{z}=\left(I-T_{u}^{\alpha}\right)_{z}=I-T_{u \circ \varphi_{z}}^{\alpha}=$ $H_{u \circ \varphi_{z}}^{\alpha}$. For $f \in A_{\alpha}^{2},\left\|H_{u \circ \varphi_{z}}^{\alpha}(f)\right\|_{2, \alpha}=\left\|\left(H_{u}^{\alpha}\right)_{z}(f)\right\|_{2, \alpha}=\left\|U_{z}^{\alpha} H_{u}^{\alpha} U_{z}^{\alpha}(f)\right\|_{2, \alpha}=$ $\left\|H_{u}^{\alpha} U_{z}^{\alpha}(f)\right\|_{2, \alpha} \leq\left\|H_{u}^{\alpha}\right\|\left\|U_{z}(f)\right\|_{2, \alpha}=\left\|H_{u}^{\alpha}\right\|\|f\|_{2, \alpha}$. Thus $\left\|H_{u \circ \varphi_{z}}^{\alpha}\right\| \leq\left\|H_{u}^{\alpha}\right\|$. Since $H_{u}^{\alpha}$ is bounded, $H_{u \circ \varphi_{z}}^{\alpha}$ is bounded.

Consider some products of Toeplitz operators and Hankel operators. The simple calculation implies that $H_{u}^{\alpha *} H_{u}^{\alpha}=T_{|u|^{2}}^{\alpha}-T_{\bar{u}}^{\alpha} T_{u}^{\alpha}$. Suppose $u \in L^{1}(\mathbb{D}, d A)$ and $f \in A_{\alpha}^{2}$. Since $H_{u}^{\alpha} f(z)=u(z) f(z)-P_{\alpha}(u f)(z)=u(z)\left\langle f, K_{z}^{\alpha}\right\rangle-\left\langle u f, K_{z}^{\alpha}\right\rangle=$ $\left\langle(u(z)-u) f, K_{z}^{\alpha}\right\rangle$ for $g \in\left(A_{\alpha}^{2}\right)^{\perp}$,

$$
\begin{aligned}
\left\langle H_{u}^{\alpha} f, g\right\rangle & =\int_{\mathbb{D}} \int_{\mathbb{D}}(u(z)-u(w)) \overline{K_{z}^{\alpha}(w)} f(w) d A_{\alpha}(w) \overline{g(z)} d A_{\alpha}(z) \\
& =\int_{\mathbb{D}} f(w) \int_{\mathbb{D}}(u(z)-u(w)) \overline{g(z)} K_{w}^{\alpha}(z) d A_{\alpha}(z) d A_{\alpha}(w) \\
& =\int_{\mathbb{D}} f(w) \overline{\left(-H_{\bar{u}}^{\alpha}\right) g(w)} d A_{\alpha}(w)=\left\langle f,-H_{\bar{u}}^{\alpha} g\right\rangle
\end{aligned}
$$

and hence $H_{u}^{\alpha *}=-H_{\bar{u}}^{\alpha}$.

Suppose $u, v, u^{2}, v^{2}$ are in $M K$. If $H_{u}^{\alpha}$ is compact, then the following are compact:
(i) $T_{|u|^{2}}^{\alpha}-T_{\bar{u}}^{\alpha} T_{u}^{\alpha}$
(ii) $T_{\bar{v} u}^{\alpha}-T_{\bar{u}}^{\alpha} T_{v}^{\alpha}$
(iii) $H_{u}^{\alpha} T_{v}^{\alpha}$
(iv) $H_{u}^{\alpha} H_{v}^{\alpha *}=H_{u}^{\alpha} H_{\bar{v}}^{\alpha}$.

Corollary 3.11. Suppose $u_{1}, \ldots, u_{n} \in M K$ and $z \in \mathbb{D}$. Then

$$
U_{z}^{\alpha} H_{u_{1}}^{\alpha} \cdots H_{u_{n}}^{\alpha} U_{z}^{\alpha}=H_{u_{1} \circ \varphi_{z}}^{\alpha} \cdots H_{u_{1} \circ \varphi_{z}}^{\alpha} .
$$

Proof. It follows from the fact that $U_{z}^{\alpha} U_{z}^{\alpha}$ is the identity operator.

Corollary 3.12. Suppose $u_{1}, u_{2} \in L^{1}(\mathbb{D}, d A)$. If $u_{1}=u_{2} \circ \varphi_{z}$ for some $z \in \mathbb{D}$, then $H_{u_{1}}^{\alpha}$ and $H_{u_{2}}^{\alpha}$ are unitary equivalent.

Proof. By Corollary 3.10, $H_{u \circ \varphi_{z}}^{\alpha}=\left(H_{u}^{\alpha}\right)_{z}$ and hence $H_{u_{1}}^{\alpha}=\left(H_{u_{2}}^{\alpha}\right)_{z}$. Thus $H_{u_{1}}^{\alpha}$ and $H_{u_{2}}^{\alpha}$ are unitary equivalent.

\section{References}

[1] S. Axler, The Bergman space, the Bloch space, and commutators of multiplication operators, Duke Math. J. 53 (1986), no. 2, 315-332.

[2] S. Axler and D. Zheng, Compact operators via the Berezin Transform, Indiana Univ. Math. 47 (1988), 387-399.

[3] A. Brown, P. R. Halmos, and A. L. Shields, Cesáro operators, Acta Sci. Math. (Szeged) 26 (1965), 125-137.

[4] K. Stroethoff, Compact Toeplitz operators on Bergman spaces, Math. Proc. Cambridge Philos. Soc. 124 (1998), no. 1, 151-160.

[5] K. Zhu, Operator Theory in Function Spaces, Marcel Dekker, Inc., New York, 1990. 
Department of Mathematics

SOOKMYUng Women's UnIVERSITY

SEOUL 140-742, Korea

E-mail address: shkang@sookmyung.ac.kr 